\title{
Multichannel QCM-based system for continuous monitoring of bacterial biofilm growth
}

Miquel-Àngel Amer, Antoni Turó, Jordi Salazar, Luis Berlanga-Herrera, Miguel J. García-Hernández, Juan A. Chávez.

\begin{abstract}
Quartz crystal microbalance (QCM) sensors are becoming a good alternative to analytical methods for the measurement of bacterial growth in liquid media culture. For this purpose, two essential resonance parameters allow monitoring of biofilm formation: the series resonance frequency shift and the change of the resistance at this frequency. Nevertheless, several problems arise in determining these parameters, as their relative variation is very small. This means that an accurate procedure must be implemented for the measurement of the QCM resonance parameters, including the automatic calibration of the frequency response effects of the measurement circuits and the automatic compensation of the static electrical capacitance of the QCM.
\end{abstract}

In this paper, a novel multichannel system for on-line monitoring of biofilm formation based on QCM sensors is proposed. QCM resonance parameters are determined from the electrical impedance analysis by means of an autobalanced impedance bridge. This configuration has allowed the implementation of an affordable multichannel measurement instrument. Obtained results, based on binary mixtures of water-glycerol measurements and real microorganism experiments, are in good agreement with the theoretical behaviour. These results show the great potential of this instrument to be used for monitoring microbial growth and biofilm formation.

\section{Introduction}

Microbiology requires systems capable of measuring, controlling and monitoring bacterial growth. For this purpose, classical methods are based on the observation of bacterial growth within liquid media cultures [1]. This is usually carried out using destructive and discontinuous techniques, which result in the breakage of the biofilm complex structure. This fact has strongly limited the study of the factors that affect the formation and control of bacteria film that take part in the experiments. For this reason, in recent years novel methods and electronic instrumentation have been devised in order to become good alternatives to these classical techniques [2]. The most relevant approaches are optical methods [3]-[6], electrical impedance measurement and spectroscopy [7]-[9], microfluidic devices [10],[11], and piezoelectric devices based systems. With respect to the latter, tuning forks [12],[13], surface acoustic wave devices [14],[15], and quartz crystal microbalances (OCM) [16]-[23], they all have demonstrated great potential in the study of biofilm growth.

In this paper, the analysis of the mechanical resonance of a QCM, on whose surface the microorganisms adhere and the biofilm grows, is used. The usefulness of QCM is that they permit the direct in situ real-time observation of the overall process, from the initial bacteria adhesion to the biofilm formation and subsequent growth, when they are compared with other popular techniques such as direct cell counting methods or the determination of dry mass. Also 
QCM techniques provide an easy way to mechanize and automate the monitoring procedure. Two essential resonance parameters reflect the changes in the QCM contacting medium: the series resonance frequency shift $\Delta f_{s}$ and the change of the resistance at this frequency $\Delta R_{m}$. These two parameters correlate with the density and viscosity of the medium [22],[23]. They are very sensitive to the changes in the solid to viscous liquid interface and to the appearance of a viscoelastic film that gradually replaces the contact of the QCM with the liquid. Nevertheless, several problems arise in the determination of $\Delta f_{s}$ and $\Delta R_{m}$ when a viscous liquid or a lossy film heavily damps the QCM as in our application happens. These constraints can be overcome by using the QCM with the widely used dissipation monitoring techniques [24],[25] or alternatively, as in the proposed system, by means of the measurement of the QCM electrical impedance [20]-[21] but addressing first several key issues that have hindered its use in this application in the past. The compensation of the static electrical capacitance $C_{0}$ of the QCM, the accurate determination of the resonance frequency in heavy damped QCM and the high frequency resolution required in order to detect small relative changes due to microorganism process are some of the most important. The current availability of high performance and low-cost analog and digital integrated circuits has made possible to implement a compact, rapid and affordable system suitable for multichannel QCM sensor measurements as requested in our application.

The novel multichannel system for on-line biofilm formation monitoring is based on QCM sensor resonance parameters obtained from the electrical impedance analysis that is measured by means of an auto-balanced impedance bridge. System architecture, measurement procedure, automatic compensation of the static electrical capacitance $C_{o}$ of the QCM, and accurate extraction of the series resonance frequency $f_{s}$ and the electrical impedance at resonance $R_{m}$ have been improved and optimised in order to meet the system demanding requirements in this application. This instrument allows the simultaneous monitoring of microbial growth and biofilm formation under controlled ambient in multiwell Petri plates by placing one QCM sensor in each well so that the measurement is only sensitive to biofilm changes. This makes it especially useful for microbiologists in the study of biofilm evolution dynamics and the research of bacterial inactivation and new biocide substances.

\section{Theoretical}

\subsection{The Butterworth Van Dyke equivalent circuit.}

A QCM is a highly sensitive sensor based on the measurement of the resonance parameters of a thickness-shear piezoelectric resonator. The sensor changes its resonance parameters due to the deposit on it of a small amount of mass [22], [23], [26]. Around its resonance, the electrical impedance of a QCM can be reduced to the Butterworth van Dyke (BVD) electrical equivalent model [27] as depicted in Fig. 1. 


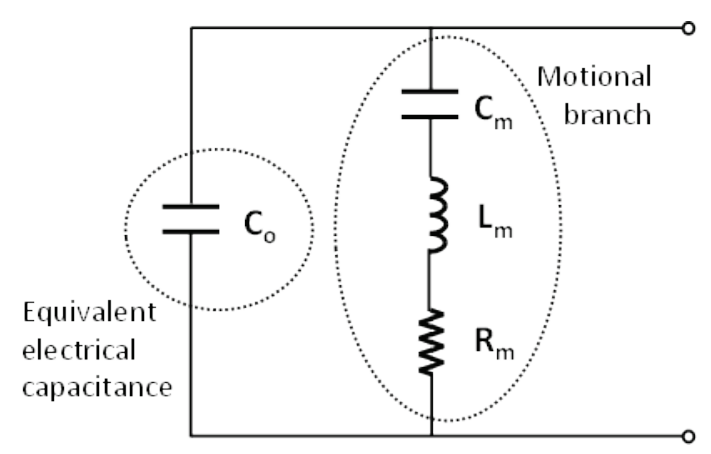

Fig. 1. Butterworth-van Dyke model of a QCM.

According to Fig. 1, the BVD circuit consists of two parallel branches. The branch on the left is the equivalent capacitance including the static electrical capacitance of the crystal, and the holder and connector capacitances. The branch on the right corresponds to the so-called motional impedance which is contributed by the acoustic load impedance acting at the surfaces of the quartz crystal. This acoustic load can consist of a single rigid film, a semiinfinite Newtonian liquid, a single viscoelastic film, or a multilayer arrangement. Therefore the total admittance can be expressed as the sum of the motional admittance and the admittance due to the capacitance $C_{o}$ as shown in (1) and the series resonance frequency is given by (2).

$$
\begin{gathered}
Y_{x}(j \omega)=j \omega C_{0}+\frac{1}{R_{m}+j\left(\omega \cdot L_{m}-\frac{1}{\omega \cdot C_{m}}\right)} \\
f_{S}=\frac{1}{2 \pi \sqrt{L_{m} \cdot C_{m}}}
\end{gathered}
$$

In a QCM application, the motional inductance $L_{m}$ increases when a mass is in contact with the crystal electrodes. This is the reason why the frequency shift of the series resonance is a sensitive indicator of the attached mass or film. Also, the motional branch can be reduced to resistance $R_{m}$ at resonance representing a maximum in the admittance. Its measurement provides important information about the acoustic load since soft films and viscous liquids increase motional losses. Nevertheless, in order to measure properly the frequency shift of the series resonance $\Delta f_{s}$ and the change of motional resistance $\Delta R_{m}$, the effect of the capacitance $C_{o}$ must be taken into account. Fig. 2 depicts, qualitatively, the typical locus of the QCM total admittance for three different values of $R_{m}$. As it can be noticed, both the high admittance zero-phase frequency $f_{r}$ and the maximum admittance frequency $f_{\text {max }}$ give a good approximation of the resonance frequency fs for low values of $R_{m}$. However, when the value of $R_{m}$ and/or the value of the parasitic capacitance $C_{o}$ increase, $f_{\max }$ and $f_{r}$ frequencies cannot be longer considered as the resonance frequency $f_{s}$. On the contrary, if the parasitic capacitance $C_{o}$ is compensated, the maximum admittance frequency $f_{\max }$ or the zero-phase frequency $f_{r}$ could be the appropriate tracking condition for the maximum admittance monitoring. 


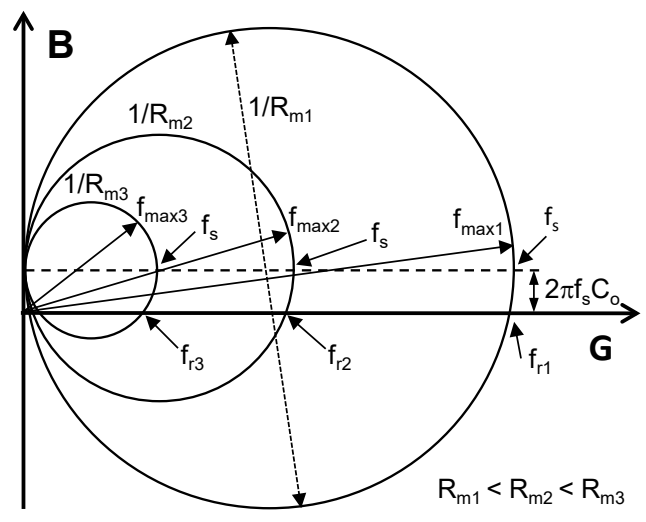

Fig. 2. Graphical representation of QCM admittance, for three different values of Rm. G and B are the real and imaginary parts of admittance.

It can be concluded that when determining fs and $\mathrm{Rm}$ from the measurement of the total QCM admittance or impedance, the capacitance effect of $C_{o}$ must be taken into account by the electronic circuit and it must be compensated accordingly.

\subsection{Liquid-contact measurements.}

The Sauerbrey expression given by (3) is commonly used to relate mass loading with resonance frequency shift $\Delta f_{s}$ for a QCM [28]. $C$ factor is the sensitivity of the resonator in $\mathrm{Hz} /\left(\mathrm{ng} / \mathrm{cm}^{2}\right)$, and $\Delta m$ is the change in mass per unit area in $\mathrm{ng} / \mathrm{cm}^{2}$. This expression is only valid for a homogeneous film rigidly attached to the QCM surface.

$$
\Delta f_{s}=-C \cdot \Delta m
$$

In the case of liquids, the operation of the QCM is also possible and its electrical impedance is sensible to the changes at the interface between the crystal and the liquid. The works carried out by Glassford [29], and later by Kanazawa and Gordon [30] demonstrated the resonance frequency dependence upon the viscosity and the density of the liquid solution in contact with the QCM. According to Kanazawa and Gordon, the change in the resonance frequency due to the liquid solution properties is given by (4).

$$
\Delta f_{s}=-f_{s}^{3 / 2}\left(\eta_{l} \rho_{l} / \pi \mu_{q} \rho_{q}\right)^{1 / 2}
$$

where $\rho_{l}$ is the density of the liquid, $\eta_{l}$ is the viscosity of the liquid, $\rho_{q}$ and $\mu_{q}$ are the density and rigidity modulus of the quartz crystal respectively.

On the other hand, viscous coupling of the liquid medium to the crystal surface results in a damped resonance oscillation. The viscous loss is manifested as an increase in resistance $R_{m}$ of the QCM resonator. Thus, the 
measurement of this resistance is a good indicator to obtain the liquid solution viscosity. The change in the resistance at series resonance of the QCM under liquid loading [31] is given by (5).

$$
\Delta R_{m}=2 n f_{s} L_{u}\left(4 \pi f_{s} \eta_{l} \rho_{l} / \mu_{q} \rho_{q}\right)^{1 / 2}
$$

Where $n$ is the number of QCM sides in contact with liquid, and $L_{u}$ is the inductance for the unperturbed, i.e. dry, resonator.

To sum up, in order to characterize the biofilm growth it is necessary to measure the two main parameters of the QCM sensor: the series resonance frequency and the resistance at this frequency.

\subsection{QCM electronic interface: design alternatives.}

In order to properly measure the QCM electrical impedance and its relevant parameters, the resonance frequency $f_{s}$ and the QCM series resistance at resonance $R_{m}$, many electronic circuits are found in literature based on network or impedance analysis [32]-[34], oscillators [35],[36], lock-in techniques [37]-[39], and impulse excitation and decay methods [40]. Most of these circuits have in common that are complex, expensive or they require manual compensation of the static capacitance $C_{o}$ effect. Moreover, these problems get even worse in the design of an online multichannel system.

Table I summarizes the advantages and disadvantages the above mentioned methods have. According to this table, the impedance measurement method gives the best results in terms of accuracy, but in the past it had serious limitations due to the high cost and large dimensions. This fact restricted its use to a laboratory environment. In addition, this option was not suitable for simultaneous multiple sensors measurements. However, nowadays the availability of high performance and low-cost analog and digital integrated circuits makes possible to implement specific impedance analysers that are compact, rapid and affordable for the accurate measurement of the QCM resonance parameters. In addition, as these circuits are optimized for fast data acquisition, they are suitable for multichannel sensor measurements as required in our application.

Finally, special attention must also be paid to the QCM interface in order to achieve the required accuracy and bandwidth. For this reason, the auto-balancing bridge method has been selected for this purpose. Also, in this work, it is proposed an automated compensation algorithm that determines the $C_{o}$ value and cancels its effect. 


\begin{tabular}{|c|c|c|}
\hline Circuit alternative & Advantages & Disadvantages \\
\hline $\begin{array}{l}\text { Impedance or Network } \\
\text { Analysers }\end{array}$ & $\begin{array}{l}\text { - No external circuitry influences. } \\
\text { - Parasitic influences excluded by } \\
\text { calibration. } \\
\text { - High accuracy measurements. }\end{array}$ & $\begin{array}{l}\text { - High cost. } \\
\text { - Complex. } \\
\text { - Large dimensions. }\end{array}$ \\
\hline $\begin{array}{l}\text { Oscillator circuit based on a } \\
\text { quartz crystal. }\end{array}$ & $\begin{array}{l}\text { - Simplicity. } \\
\text { - Low cost. } \\
\text { - Continuous and rapid monitoring of } \\
\text { the resonance frequency. }\end{array}$ & $\begin{array}{l}\text { - Resonance frequency and resistance at } \\
\text { resonance frequency measurements are } \\
\text { affected by capacitance } C_{o} \text {. } \\
\text { - Difficulty in maintaining the circuit } \\
\text { oscillation due to parasitic capacitances. } \\
\text { - Manual procedure to compensate } \\
\text { capacitor } C_{o} \text {. }\end{array}$ \\
\hline Phase-looked-loop circuit & $\begin{array}{l}\text { - Relatively simple circuit. } \\
\text { - Capacitance } C_{o} \text { compensation. } \\
\text { - Continuous monitoring of the } \\
\text { resonance frequency and resistance at } \\
\text { series resonance. }\end{array}$ & $\begin{array}{l}\text { - Complex calibration if a good } \\
\text { compensation of capacitance } C_{o} \text { is } \\
\text { required. } \\
\text { - Difficulty to automate the compensation } \\
\text { of capacitance. }\end{array}$ \\
\hline $\begin{array}{l}\text { Impulse excitation and } \\
\text { decay methods. }\end{array}$ & $\begin{array}{l}\text { - Accurate measurement of resonance } \\
\text { frequency. } \\
\text { - Easy cancellation of } C_{o} \text {. }\end{array}$ & $\begin{array}{l}\text { - More appropriate for laboratory } \\
\text { environments. } \\
\text { - High complexity and cost for accurate } \\
\text { measurements of highly damped QCM. }\end{array}$ \\
\hline
\end{tabular}

Table I. Circuit alternatives for resonance frequency and resistance at series resonance measurements: advantages and disadvantages.

\section{Fundamentals of the measurement system.}

\subsection{Auto-balancing impedance bridge.}

An auto-balancing impedance bridge based circuit has been chosen for implementing the QCM interface in the measurement system. This technique is exceptionally accurate over a broad range of impedances and frequencies and it has the advantage of maintaining one of the QCM terminals virtually connected to ground.

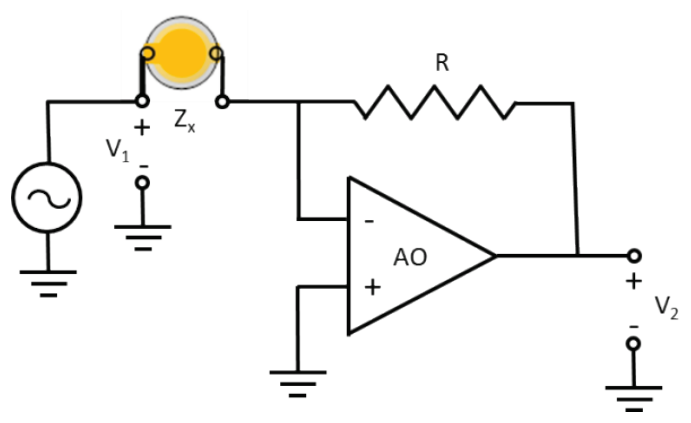

Fig. 3. Auto-balancing impedance bridge scheme. 
As shown in Fig. 3, the auto-balancing impedance bridge is based on an I-V converter configured as an inverting op-amp stage. Basically, it employs a feedback resistor $\mathrm{R}$, the unknown impedance $Z_{X}$ that corresponds to the QCM sensor impedance, and a reference voltage source $V_{1}$. Applying the Kirchoff's Current Law, it is possible to calculate the QCM sensor electrical impedance $Z_{x}$ at the angular frequency $\omega$ of the reference voltage source from the circuit parameters by means of (6).

$$
Z_{x}(j \omega) \cong-\frac{V_{1}(j \omega)}{V_{2}(j \omega)} \cdot R
$$

From (6), the magnitude and phase of the QCM sensor impedance at a specific frequency $\omega$ can be calculated. In order to find the resonance parameters of the sensor, the frequency shift of the series resonance $\Delta f_{s}$ and the change of the motional resistance $\Delta R_{m}$, a frequency sweep of the reference voltage source is performed. Therefore, the impedance $Z_{x}$ is measured at different frequencies in order to find its minimum, which corresponds to the resonance.

It should be noted that the impedance measured from the circuit described in Fig. 3 corresponds to the total impedance $Z_{x}(j \omega)$, that is composed of the motional impedance $Z_{m}(j \omega)$ and the impedance due to the electrical capacitance $Z_{c o}(j \omega)$. For this reason, in order to achieve a better accuracy in the measurement of the resonance parameters, the parasitic capacitance $C_{o}$ must be compensated.

\subsection{Compensation of the op-amp frequency response effect}

The selection of the op-amp is critical as the accuracy at high frequencies is affected by the open-loop voltage gain frequency response of the op-amp used in the I-V converter. For this reason, an op-amp with a high GBW must be chosen in order to reduce the measurement error at high frequencies.

In order to calculate the impedance $Z_{x}(j \omega)$, both signals $V_{1}(j \omega)$ and $V_{2}(j \omega)$ must be measured as depicted in (6). Due to the fact that the $V_{2}(j \omega)$ value is affected by the frequency response of the op-amp, the measured value does not correspond with the real one. The equation (7) gives the relationship between both values.

$$
Z_{x e}(j \omega)=\frac{V_{2}(j \omega)}{V_{1}(j \omega)}=Z_{x}(j \omega)+\frac{R+Z_{x}(j \omega)}{a(j \omega)}
$$

Where $Z_{x e}(j \omega)$ corresponds to the estimated value of the impedance, obtained from the measurements of the signals $V_{1}(j \omega)$ and $V_{2}(j \omega)$ by using the auto-balanced bridge, $Z_{x}(j \omega)$ corresponds to the real value of the impedance, and $a(j \omega)$ is the op-amp frequency response.

In order to reduce the error introduced by the op-amp frequency response, a calibration procedure was developed, which consists of measuring two different known impedance values by using the auto-balancing impedance bridge: a 
short-circuit and a known accurate impedance, preferably a resistance, to minimise errors. Consequently, the electrical impedance of the $\mathrm{QCM} Z_{x}(j \omega)$ can be obtained from these calibration measurements by means of (8)

$$
Z_{x}(j \omega)=Z_{c a l}(j \omega) \cdot \frac{Z_{x e}(j \omega)-Z_{c c e}(j \omega)}{Z_{c a l e}(j \omega)-Z_{c c e}(j \omega)}
$$

In (8), $Z_{c c e}(j \omega)$ and $Z_{\text {cale }}(j \omega)$ correspond to the estimated values of the QCM impedance for the short-circuit and the known accurate impedance, respectively. $Z_{c a l}(j \omega)$ is the real value of the known impedance.

\subsection{Capacitance $C_{0}$ compensation algorithm.}

As explained in section 2.1, the effect of the QCM static capacitance $C_{o}$ must be cancelled from the measurement of electrical impedance $Z_{x}(j \omega)$. The simplest way is to estimate its value from impedance measurements and then use it for the cancellation from the total impedance $Z_{x}(j \omega)$. For this purpose, the motional impedance $Z_{m}(j \omega)$ and the impedance due to the capacitance $Z_{c o}(j \omega)$ must be taken into account, as shown in (9).

$$
Z_{m}(j \omega)=\frac{Z_{x}(j \omega) \cdot Z_{C o}(j \omega)}{Z_{C o}(j \omega)-Z_{x}(j \omega)}
$$

The estimation of capacitance $C_{o}$ is performed at a frequency $\omega_{\mathrm{a}}$ much lower than the series resonance frequency $\omega_{\mathrm{s}}$, where the QCM total impedance $Z_{x}\left(j \omega_{a}\right)$ is approximately equal to the impedance due to the electrical capacitance $Z_{c o}\left(j \omega_{a}\right)$ as given by $(10)$.

$$
Z_{x}\left(j \omega_{a}\right) \cong Z_{C o}\left(j \omega_{a}\right)=\frac{1}{j \cdot \operatorname{Co} \cdot \omega_{a}} \quad\left(\omega_{a} \ll \omega_{s}\right)
$$

Then, the impedance $Z_{c o}(j \omega)$ at any frequency $\omega$ is obtained by means of (11). This makes possible to determine the impedance of the motional branch $Z_{m}(j \omega)$ from the measurement of the total impedance $Z_{x}(j \omega)$ by means of the compensation of the effect of $C_{o}$ by using (9).

$$
Z_{C o}(j \omega) \cong \frac{\omega_{a}}{\omega} \cdot Z_{C o}\left(j \omega_{a}\right)
$$

\subsection{Sinusoidal signal characterization of $V_{1}$ and $V_{2}$ : sine wave fitting algorithm.}

The estimation of QCM electrical impedance $Z_{x e}(j \omega)$ is obtained by measuring the excitation voltage $V_{1}(j \omega)$, and the output voltage $V_{2}(j \omega)$ at different frequencies, as shown in (6). Therefore, obtaining an accurate impedance value depends directly on the precise measurement of the amplitude and phase of these voltage signals. Moreover, this measurement is especially difficult near the resonance frequency where the signal amplitudes can be very small. 
There are many methods to estimate the parameters of a sinusoidal digitized signal, i.e., amplitude, phase, frequency and DC component [41]-[44]. Sedlack et al. [45] compare these methods and concluded that the sinewave-fit methods are the best ones from the point of view of both measurement bias and uncertainty. The sinewave-fit technique is based on the least-square error (LSE) between the measured signal and an ideal sinusoid. There are standardized algorithms for the estimation of three parameters [46] (amplitude, frequency and phase) and four parameters [47] (amplitude, frequency, phase and DC component).

With reference to the 3-parameter algorithm, the frequency estimation is inaccurate and the DC component is not taken into account. The 4-parameter method overcomes these limitations, however, if applied separately on two different signals, the 4-parameter method will result in the estimation of two different frequencies, despite the fact that the two records are from signals of the same frequency. Some authors [48] suggested to solve this problem by applying the new 7-parameter sine-fitting algorithm (7PSF). This algorithm uses both records to determine all parameters of sine waves. If the 7PSF algorithm is applied, then only estimated frequency is obtained for both signals, $V_{1}$ and $V_{2}$. This is the major advantage of this method. Also, obtained results have shown that the sine-fitting method compared to the zero-crossing and peak detection methods achieves better results without an excessive associated computational burden.

So, according to the 7PSF algorithm, the signals to be acquired are:

$$
\begin{aligned}
& V_{1}(t)=V_{10} \sin \left(2 \pi f t+\varphi_{1}\right)+V_{1 o f f s e t} \\
& V_{2}(t)=V_{20} \sin \left(2 \pi f t+\varphi_{2}\right)+V_{2 o f f s e t}
\end{aligned}
$$

where $f$ is the frequency, $V_{i 0}$ is the amplitude, $V_{\text {ioffset }}$ is the DC component and $\phi_{i}$ is the phase angle, for $i$ equal to 1 and 2.

Both expressions can be expanded as

$$
\begin{aligned}
& V_{1}(t)=A_{1} \sin (2 \pi f t)+B_{1} \cos (2 \pi f t)+C_{1} \\
& V_{2}(t)=A_{2} \sin (2 \pi f t)+B_{2} \cos (2 \pi f t)+C_{2}
\end{aligned}
$$

being $A_{i}, B_{i}$ and $C_{i}$ are constant components that can be related to $V_{i 0}, \phi_{i}$, and $V_{i o f f s e t}$.

The two sine signals, $V_{1}(t)$ and $V_{2}(t)$ are equally-spaced sampled. The first $M$ samples of the first channel are: $y_{11}$, $y_{12}, \ldots, y_{1 M}$. The same number of samples for the second channel are: $y_{21}, y_{22}, \ldots, y_{2 m}$. When $M$ is higher than 4 the system is overdetermined and the least square method is applied in order to obtain a unique solution for all the parameters $A_{i}, B_{i}, C_{i}$ and $f$. The proposed method [49] obtains these parameters by minimizing the residual leastsquare error $\varepsilon$ as given by (14). 


$$
\begin{aligned}
\varepsilon & =\sum_{n=1}^{M}\left[y_{1 n}-A_{1 k} \sin \left(2 \pi f t_{n}\right)-B_{1 k} \cos \left(2 \pi f t_{n}\right)-C_{1 k}\right]^{2} \\
& +\sum_{n=1}^{M}\left[y_{2 n}-A_{2 k} \sin \left(2 \pi f t_{n}\right)-B_{2 k} \cos \left(2 \pi f t_{n}\right)-C_{2 k}\right]^{2}
\end{aligned}
$$

Where $k$ is the iteration index. As a final result we get a unique constant set $\left\{A_{1 k}, B_{1 k}, C_{1 k}, f, A_{2 k}, B_{2 k}, C_{2 k}\right\}$.

\section{Description of the measurement system.}

\subsection{Block diagram of the measurement system.}

The measurement system has been designed to monitor the impedance changes of an array of QCM sensors when they are placed inside of a multi-well Petri plate with liquid samples. Results are presented and stored in a personal computer. A graphic user interface permits to easily control and configure the measurement tests and to store the results in a file.

Fig. 4 shows the system architecture. It consists of four blocks in order to provide high versatility: the sensor interface \& analog front end, the data acquisition \& communication block, the signal generation block and the control block. The architecture is very flexible and fully scalable. Therefore, new measurement channels can be easily added by expanding the data acquisition and signal generation blocks. Also, the cost of the whole system can be drastically reduced by multiplexing the inputs of the acquisition block and the outputs of the signal generation block if the measurement speed requirement is not too restrictive.

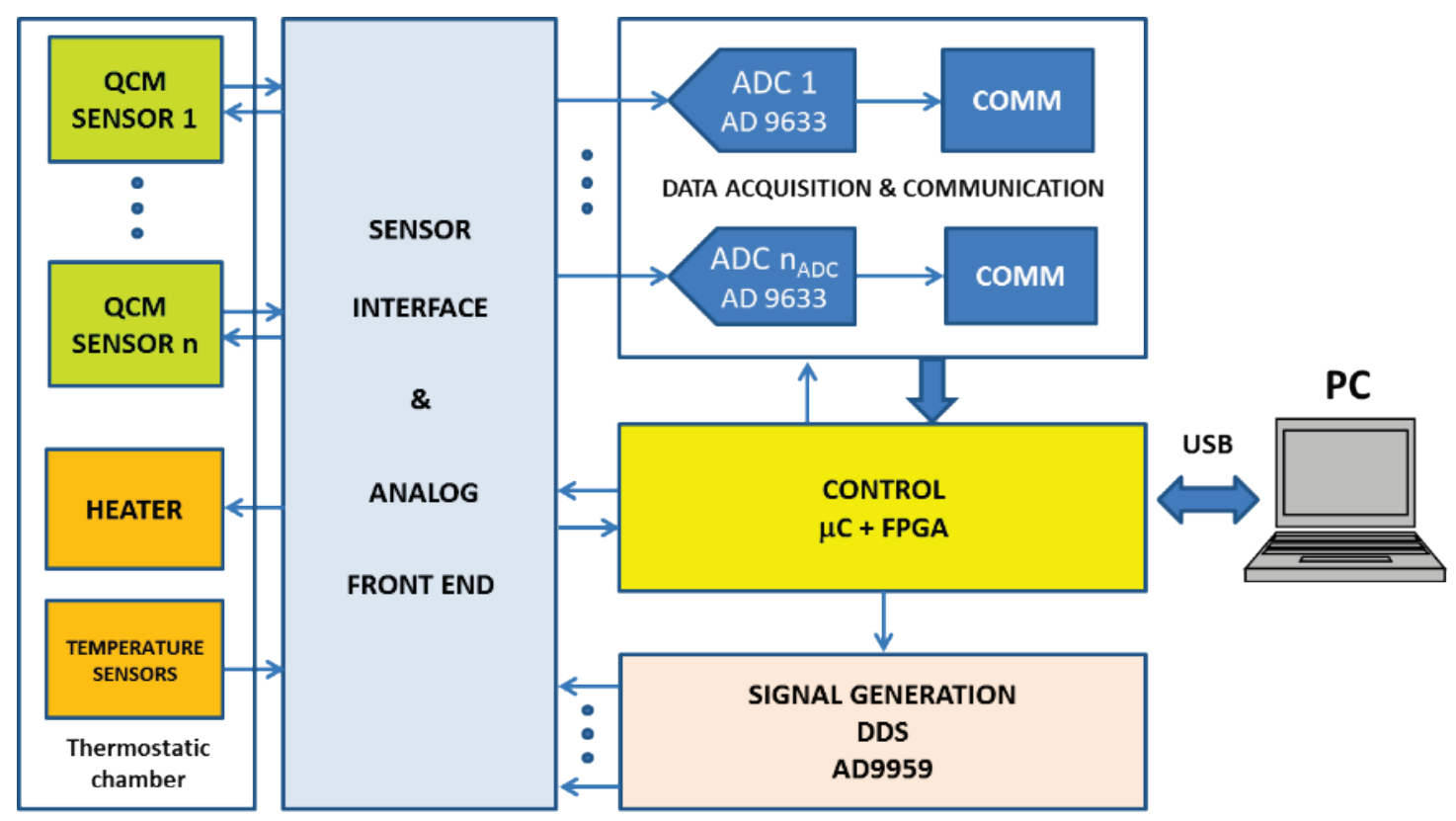

Fig. 4. Block diagram of the measurement system. 


\subsection{Sensor interface and analog front end.}

This block includes the QCM interface and signal conditioning circuits that are responsible for properly obtaining the voltage and current of each QCM sensor in order to calculate its impedance at every excitation frequency. Fig. 5 shows the electrical circuit schematics for each measurement channel.

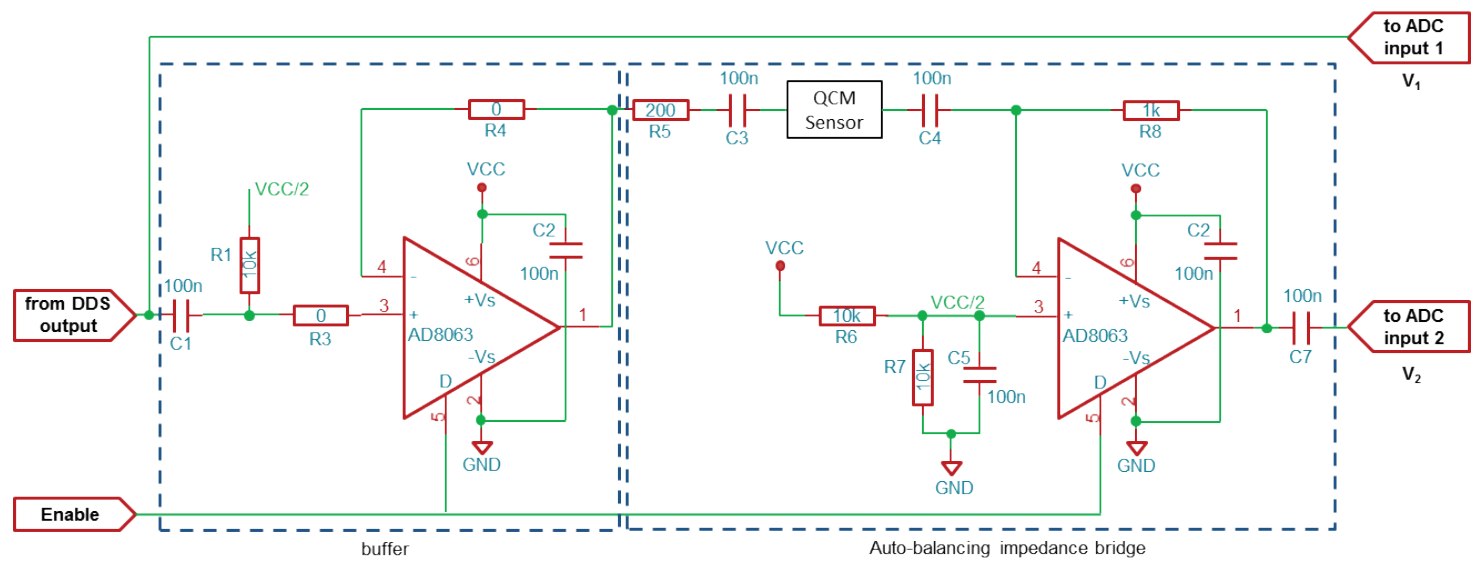

Fig. 5. Sensor interface and signal conditioning circuit.

As explained in section 3.1, this circuit is based on an auto-balancing impedance bridge in order to obtain the electrical impedance of the device by means of (6). The voltage $V_{1}$ is generated by a DDS and then applied to one of the terminals of the QCM sensor by means of a buffer stage (op-amp on the left) in order to prevent loading effects and to avoid interfering the desired operation. The other QCM terminal is virtually connected to ground by means of the op-amp on the right. This second op-amp also converts the QCM current into an equivalent voltage, $V_{2}$. The use of AD8063 operational amplifier features a large gain-bandwidth product that makes it suitable for our application. In addition, it has an input that enables or disables each measurement channel if required by putting the output at high impedance state. The system selects which measurement channel is active by enabling its corresponding pin and puts the rest of channel outputs at high impedance. The control of the enable pin of the different op-amps allows multiplexing several measurement inputs with a single acquisition channel and avoiding the use of an analog multiplexer or analog switches.

\subsection{Signal generation.}

A Direct Signal Synthesizer (DDS) is used to generate the frequency-sweep sinusoidal signal to perform the impedance measurement in the frequency range of interest. The DDS architecture allows a simple and low-cost implementation of an arbitrary waveform generator. The AD9959 synthesizer from Analog Devices has been used for this purpose. It is composed of four different channels which permit to perform several measurements in parallel. 
Each channel is able to generate an output signal with a frequency up to $200 \mathrm{MHz}$ with a resolution of $0.23 \mathrm{~Hz}$, which is much more than what is required by the system.

\subsection{Data acquisition \& communication.}

Two A/D converters are used to capture the analog signals corresponding to the voltage and the current of the QCM sensor for each auto-balancing impedance bridge, i.e., each measurement channel. Depending on the number of measurement channels and the measurement speed requirements, several channels can be multiplexed in order to use only a single A/D converter for them. The AD9633 A/D converter from Analog Devices has been chosen. This is a 12-bit converter with a sampling rate up to 125 MSPS. As the QCM sensor resonance frequencies are within the range from 5 to $10 \mathrm{MHz}$, its features permit to accurately acquire the signal parameters, and then to calculate the amplitude and phase of the impedance at each frequency. The blocks of digital data from the A/D converters are transferred to different FIFO included in a Xilinx Virtex-4 FPGA device. Finally, data is sent to the PC through the USB communication bus.

\subsection{Control.}

Three main tasks must be controlled in order to perform the impedance measurements: the enable and disable of the QCM sensor input channels, the configuration of the DDS device in order to set up the signal generator, and the configuration of the A/D conversion and the supervision of data communication. A PIC32 microcontroller from Microchip carries out the first two tasks and the later one is assigned to the Xilinx Virtex-4 FPGA.

In addition, the temperature control of the measurement environment is a critical issue during the monitoring of biofilm growth. For this reason, the multi-well Petri plate with the QCM sensors is placed into a thermostatic chamber. Three different NTC temperature sensors are used to monitor the temperature at three different locations. Each temperature sensor has a conditioning circuit that linearizes and prepares the measurement in order to be acquired with the internal A/D converters of the PIC32 microcontroller. The control of the temperature in the thermostatic chamber is achieved by means of the activation/deactivation of a heater controlled from the microcontroller.

\subsection{Calibration and measurement procedure.}

As explained in section 3, raw data obtained from the acquisition of voltage and current at the device under test must be processed accordingly in order to get good results in the QCM resonance frequency and resistance at resonance. The first step is the use of a 7PSF-algorithm to extract the amplitude, phase, DC component and frequency of the voltage and current signals. The frequency is also obtained from the measurements because it 
provides a better accuracy than when using the frequency input parameter of the DDS. The measured impedance using the self-balance impedance bridge includes some parasitic components, such as the DC blocking capacitors or the resistance R1. In addition, there is an error due to the non-ideal behaviour of the operational amplifiers. In order to minimise all these effects, a calibration procedure has been developed.

The accuracy in the extraction of the impedance resonance parameters depends on the cancellation of the electrical capacitance $C_{0}$ of the QCM sensor. The estimation of the value of $C_{0}$ is performed by means of the impedance measurement at a frequency far below the resonance frequency where the impedance is only contributed by this capacitance, as explained in section 3.3. Once the capacitor value has been estimated, its effect is subtracted from the measured impedance, leaving only the impedance of the QCM motional branch.

Finally, an iterative procedure, as depicted in Fig. 6, has been developed to determine the QCM sensor parameters at series resonance: resonance frequency $f_{s}$ and impedance at resonance $R_{m}$ in both magnitude and phase. In order to reduce the measurement time per channel, the frequency range is progressively narrowed around the resonance at each iteration while maintaining the number of steps in the frequency sweep. First sweeps are needed to know where the resonance is approximately, while the following sweeps are limited to the frequency range around the resonance, so improving the accuracy. This procedure reduces the computational burden of the system due to the number of measurements and calculations carried out, what permits the continuous monitoring of resonance parameters in multiple channels.

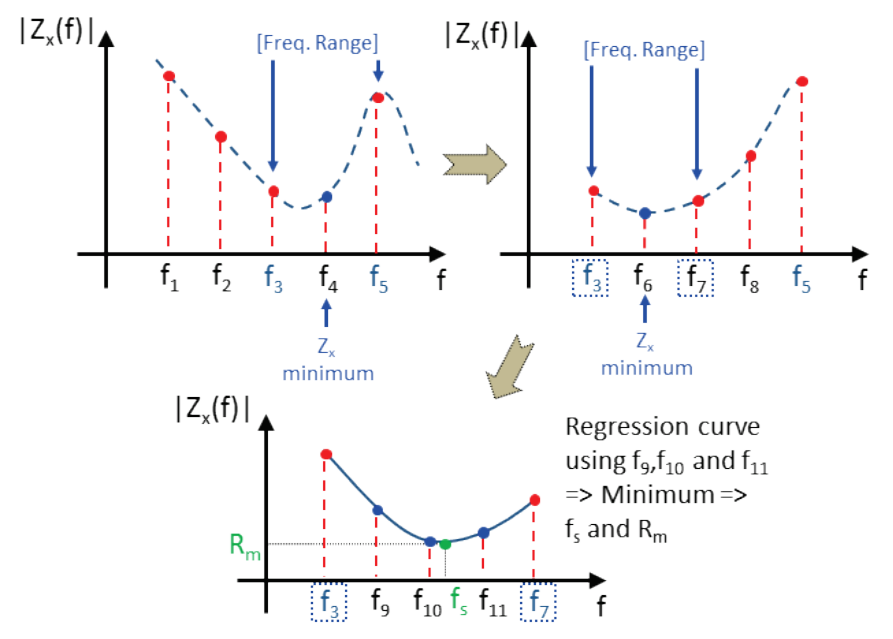

Fig. 6. Procedure for the determination of the QCM resonance parameters.

After five iterations, five points around the resonance are determined. Then, measurements of the impedance magnitude and phase in the same five points are repeated and are averaged. From the measurements averaged previously, regression curves are fitted. In addition, the mean square error is determined and compared with a threshold in order to validate the results obtained. For the impedance magnitude, a second order polynomial is used to perform a parabolic fitting. For the impedance phase, a first order polynomial is used to perform a straight fitting. Then, the minimum of impedance is found and the corresponding phase and frequency are also determined. These 
values correspond to the resonance parameters: resonance frequency and impedance magnitude and phase at resonance. A flow chart of the measurement procedure is shown in Fig. 7.

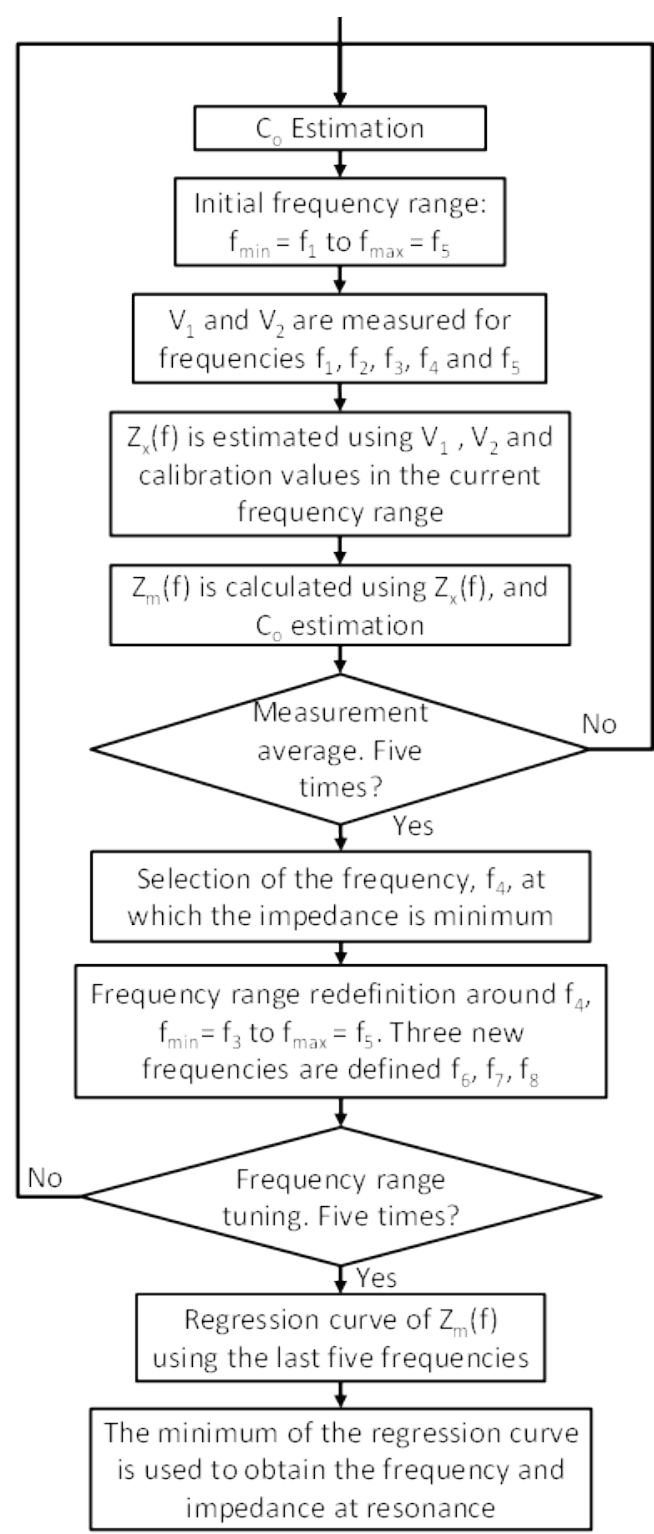

Fig. 7. Flow chart of the measurement procedure.

\subsection{Software.}

A software application has been developed in LabVIEW in order to provide the graphical user interface and to control the measurement system. The program runs on a personal computer that communicates with the system via USB. The graphical user interface permits the user to interact with the system setting the main parameters of the experiments as shown in Fig. 8: number of measurement channels, total measurement duration, time step, frequency range and thermostatic chamber temperature. It also permits to perform the calibration of the system. Once the user selects the experiment parameters, this information is sent to the control unit of the measurement system that adjusts the setting of the ADC and DDS modules. The results of the experiment are shown in real time for each channel as in Fig. 8. 

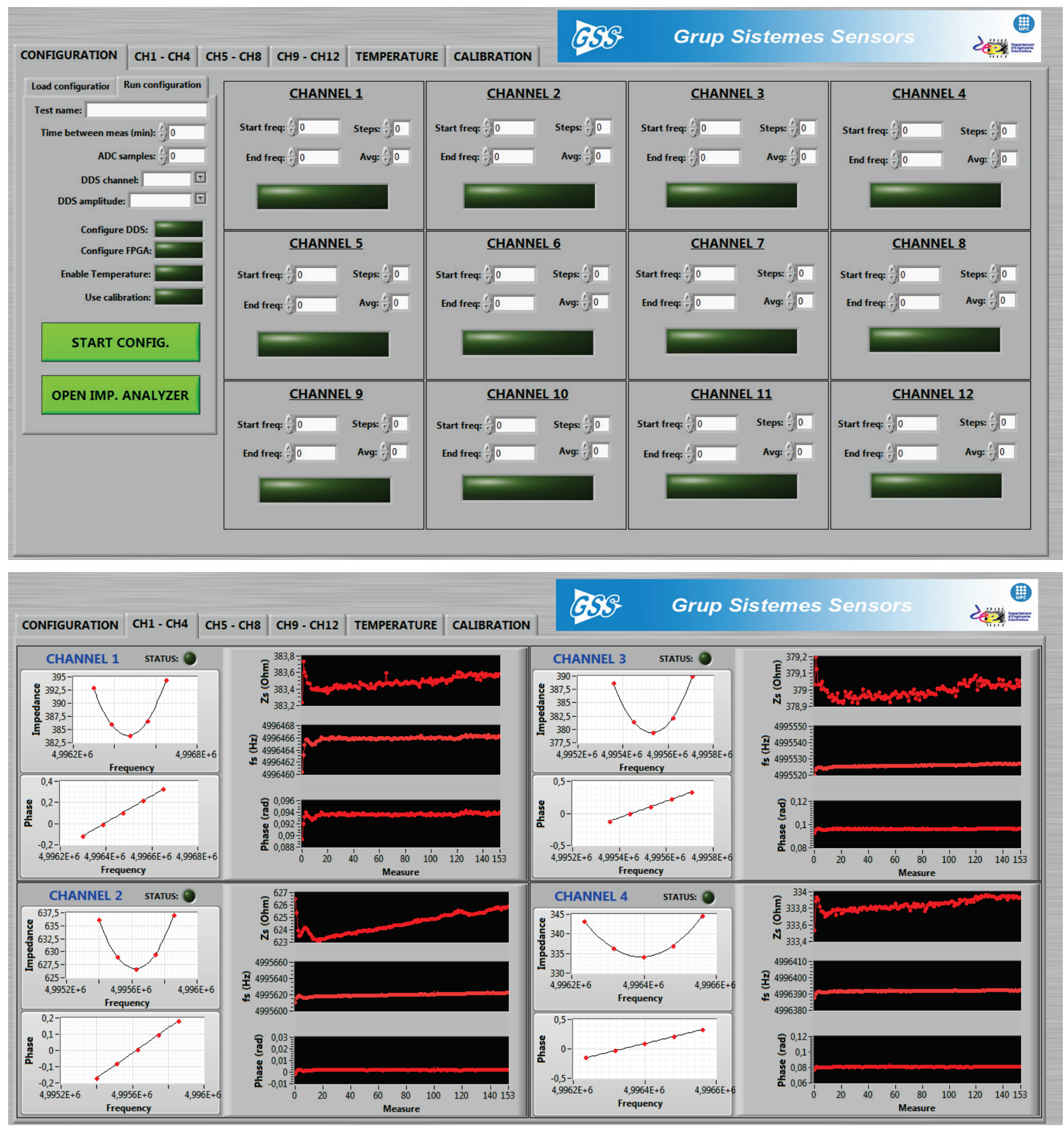

Fig. 8. Graphical user interface: configuration (up) and results (bottom) windows.

\section{Results.}

In order to validate the correct operation of the developed multichannel system, the QCM series resonance frequency and resistance at resonance was measured with the QCM sensor in contact with three different media: air, different glycerol aqueous solutions and, the last one, a culture with microorganisms in order to monitor qualitatively the biofilm growth. 

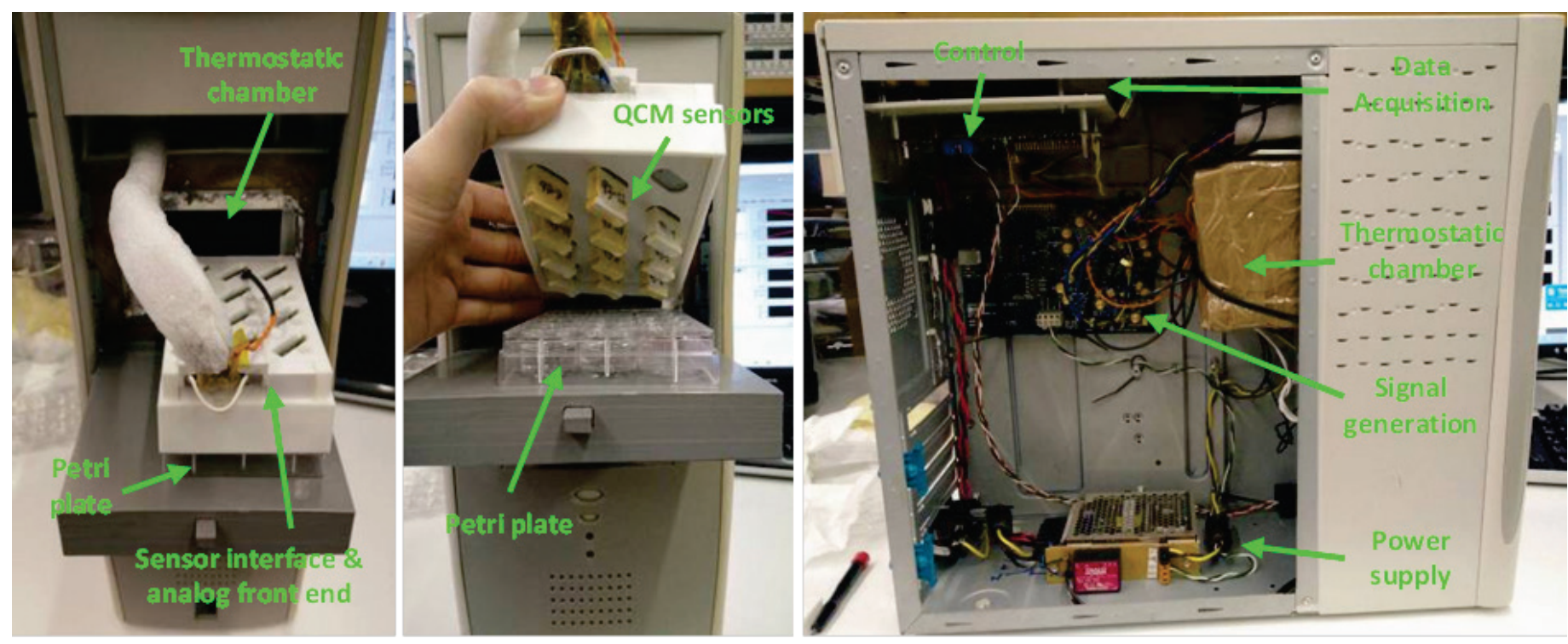

Fig. 9. Experimental prototype of the system.

A 1/2" QCM crystal manufactured from ICM (International Crystal Manufacturing, ref. 151527-5) was chosen to perform the experimental measurements. The AT-cut quartz crystal discs have a resonance frequency of $5 \mathrm{MHz}, \mathrm{a}$ resistance at resonance of $10 \Omega$ in air and gold plated titanium electrodes (Fig. 10a).
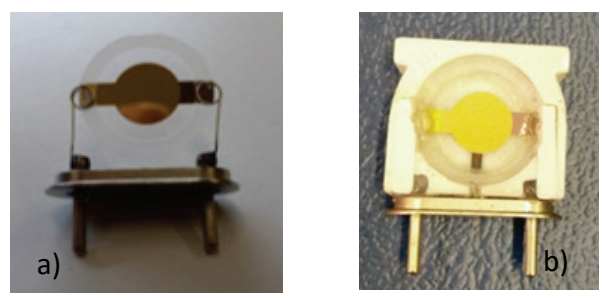

Fig. 10. Image of the ICM $1 / 2$ " QCM crystal (a) and ABS holder (b)

For a correct use of the QCM sensors, the crystals must be mounted in holders in such a way that only one of the crystal sides is exposed to the medium to be measured, and the other one is maintained isolated (Fig. 10b). In our application, the use of traditional commercial holders is not suitable as they are too big and expensive. Our holder design is based on one-piece compact construction that houses the quartz crystal maintaining the back side without any contact or acoustic loading. Silicone paste is used to provide the required sealing to prevent contact between the internal side of the crystal and the medium. The holder is made of ABS (Acrylonitrile Butadiene Styrene) polymer, by means of a 3D printer. This holder design provides an important advantage with respect commercial holders because it allows our QCM sensors to be immersed into the microorganism culture in a vertical position. Thus, unlike an horizontal position at the cell bottom, only the effect of the biofilm attached to the electrode is measured and not the material deposited by gravity on the culture cell bottom [50]. 


\subsection{Resonance measurements of QCM sensors in air.}

Measurements of the QCM sensor resonance parameters in air have been performed in order to verify the proper operation of the implemented measurement system and to determine its accuracy. Results have been compared with the ones obtained by using a commercial Agilent 4294A precision impedance analyser. The Agilent 4294A impedance analyser performs precise electrical impedance measurements in the frequency range from $40 \mathrm{~Hz}$ to $110 \mathrm{MHz}$ with a resolution of $1 \mathrm{mHz}$. Its specified four-terminal impedance measurement accuracy ranges from $0.1 \%$ to $1 \%$ in the ranges of interest in frequency (from 1 to $10 \mathrm{MHz}$ ) and impedance (from $1 \Omega$ to $10 \mathrm{k} \Omega$ ) for our application. The measurement range of the developed system is from 1 to $10 \mathrm{MHZ}$ but it could be extended until 200 $\mathrm{MHz}$ with a resolution of $0.23 \mathrm{~Hz}$ and the impedance accuracy is $2 \%$ in the measurement range taking the $4294 \mathrm{~A}$ analyser as reference.

Table II and Table III present the series resonance frequency and the resistance at resonance measurements of a set of eleven QCM sensors placed in a twelve empty well Petri plate. The remaining well is used for reference purposes. Sensors have been placed inside a thermostatic chamber at $37^{\circ} \mathrm{C}$ in order to achieve more stable measurements.

\begin{tabular}{|c|c|c|c|c|}
\hline \multirow{2}{*}{$\begin{array}{l}\text { QCM sensor } \\
\text { number }\end{array}$} & \multicolumn{2}{|c|}{ Resonance frequency $f_{s}(\mathrm{MHz})$} & \multirow{2}{*}{$\begin{array}{l}\text { Absolute error } \\
\qquad(\mathrm{Hz})\end{array}$} & \multirow{2}{*}{$\begin{array}{c}\text { Relative error } \\
\text { (\%) }\end{array}$} \\
\hline & Agilent 4294A & $\begin{array}{c}\text { Developed } \\
\text { system }\end{array}$ & & \\
\hline 1 & 4.9965 & 4.996545 & 45 & 0.0009 \\
\hline 2 & 4.9956 & 4.995822 & 222 & 0.0050 \\
\hline 3 & 4.9956 & 4.995615 & 15 & 0.0003 \\
\hline 4 & 4.9962 & 4.996396 & 196 & 0.0040 \\
\hline 5 & 4.9938 & 4.993920 & 120 & 0.0030 \\
\hline 6 & 4.9938 & 4.993958 & 158 & 0.0040 \\
\hline 7 & 4.9959 & 4.996123 & 223 & 0.0050 \\
\hline 8 & 4.9932 & 4.993374 & 174 & 0.0040 \\
\hline 9 & 4.9947 & 4.994907 & 207 & 0.0050 \\
\hline 10 & 4.9929 & 4.992931 & 31 & 0.0007 \\
\hline 11 & 4.9926 & 4.992726 & 126 & 0.0030 \\
\hline
\end{tabular}

Table II. Series resonance frequency measurements of the QCM sensors in air at $37^{\circ} \mathrm{C}$. 


\begin{tabular}{|c|r|r|r|c|}
\hline \multirow{2}{*}{$\begin{array}{c}\text { QCM sensor } \\
\text { number }\end{array}$} & \multicolumn{2}{|c|}{$R_{m}(\Omega)$} & $\begin{array}{c}\text { Series resonance resistance } \\
(\Omega)\end{array}$ & $\begin{array}{c}\text { Relative error } \\
(\%)\end{array}$ \\
\cline { 2 - 5 } & Agilent 4294A & $\begin{array}{c}\text { Developed } \\
\text { system }\end{array}$ & & 0.05 \\
\hline 1 & 416.2 & 416.0 & 0.2 & 0.20 \\
\hline 2 & 618.7 & 617.9 & 0.8 & 1.00 \\
\hline 3 & 383.4 & 379.7 & 3.7 & 2.00 \\
\hline 4 & 342.4 & 338.3 & 4.1 & 0.40 \\
\hline 5 & 548.4 & 550.6 & 2.2 & 0.80 \\
\hline 6 & 446.2 & 442.8 & 3.4 & 0.70 \\
\hline 7 & 791.4 & 785.8 & 5.6 & 0.90 \\
\hline 8 & 772.4 & 765.6 & 6.8 & 2.00 \\
\hline 9 & 308.8 & 302.6 & 6.2 & 2.00 \\
\hline 10 & 498.6 & 492.3 & 6.3 & 0.07 \\
\hline 11 & 708.3 & 707.8 & 0.5 & \\
\hline & & & & \\
\hline
\end{tabular}

Table III. Resistance at resonance measurements of the QCM sensors in air at $37^{\circ} \mathrm{C}$.

Table II shows series resonance frequency results obtained with Agilent 4294A impedance analyser and the developed multichannel system. Comparing obtained results, no significant differences between both systems are observed and the relative error is less than $0.005 \%$.

Similarly, Table III compares resistance at resonance measurements obtained with the Agilent 4294A impedance analyser and the developed multichannel system. In this case, the relative error between both systems is less than $2 \%$ for the worst case.

\subsection{Resonance measurements of QCM sensors immersed in different glycerol aqueous solutions}

Different aqueous solutions of glycerol at different concentrations (0\%, 10\%, $20 \%, 30 \%, 40 \%, 50 \%$ and $60 \%$ ) have been prepared in order to quantify variations of the QCM sensor resonance parameters in different liquid media. The objective is to demonstrate the effectiveness of the system when measuring QCM resonance parameters when they are loaded by high viscous media as occurs in our application. Then, for every different solution, ten wells of a Petri plate were filled with the same amount $(3.8 \mathrm{ml})$ of glycerol solution, and a QCM sensor was immersed in every well of the Petri plate. The whole system was placed inside a thermostatic chamber at $38^{\circ} \mathrm{C}$ and, once temperature stability was reached, the resonance parameters of every sensor were measured. 
Fig. 11 shows the series resonance frequency shift measurements in binary mixtures of water-glycerol at different concentrations of one of the QCM sensors. Results were compared with the theoretical ones provided by the Kanazawa equation.

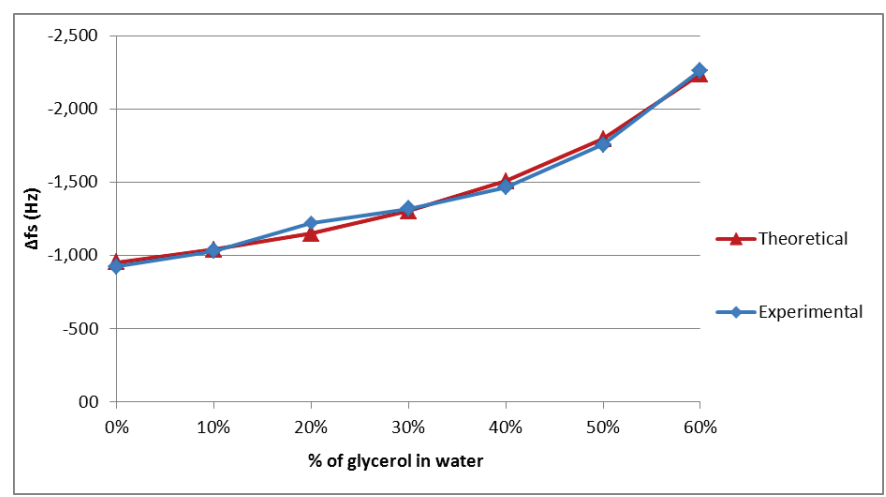

Fig. 11. Series resonance frequency shift in binary mixtures of water-glycerol thermostated to $38^{\circ} \mathrm{C}$. Red line corresponds to the values obtained by applying the Kanazawa theoretical equation. Blue line corresponds to the measured results.

From data represented in Fig. 11, it is possible to calculate the accuracy of the series resonance frequency measurements, related to the expected values according to the Kanazawa theoretical equation. In this case, the relative error is less than $3 \%$ for the worst case. This proves that experimental results obtained are in good agreement with the theoretical behaviour and it demonstrates the effectiveness of the $C_{o}$ compensation.

Similarly, Fig. 12 shows the resistance at resonance shift measurements in binary mixtures of water-glycerol at different concentrations, for the same QCM sensor. Again, results obtained are in good agreement with the theoretical behaviour provided by the Kanazawa equation.

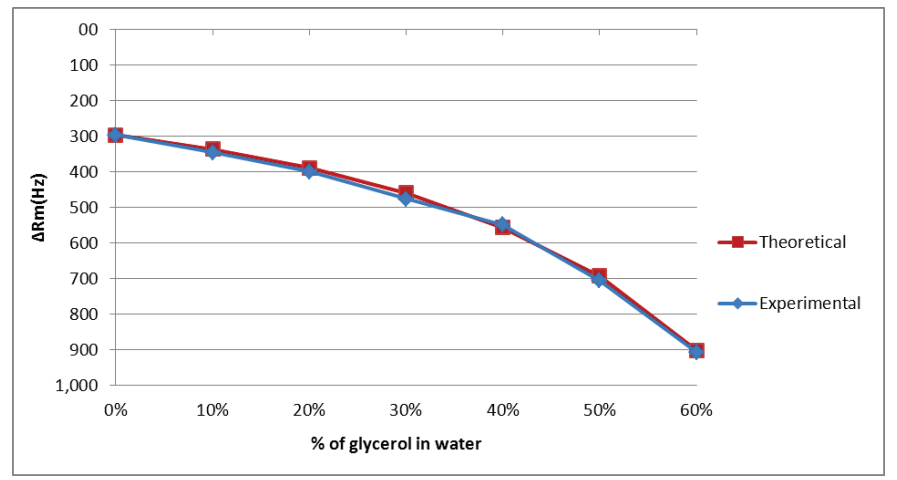

Fig. 12 Resistance at resonance shift in binary mixtures of water-glycerol thermostated to $38^{\circ} \mathrm{C}$. Red line corresponds to the values obtained by applying the Kanazawa theoretical equation. Blue line corresponds to the measured results.

Again, from data represented in Fig. 12, it is possible to calculate the accuracy of the resistance at resonance measurements, related to the expected values according to the Kanazawa theoretical equation. According this, the relative error is about 3.5\% for the worst case, which proves the good agreement with the theoretical values. 
These results demonstrate that the system properly compensates the effect of the viscous losses in the extraction of the impedance resonance parameters.

\subsection{Biofilm formation monitoring.}

Finally, an experiment with real microorganisms has been carried out in order to perform a qualitative measurement of the biofilm growth. For this purpose, four wells of the measurement system were filled with a culture containing the microorganisms and a QCM sensor was immersed in every well. The bacteria chosen for the experiment was the Staphylococcus epidermidis. The selection of this type of bacteria was due to its regular presence in the environment, the ability to quickly form a robust biofilm that make it suitable for the experimentation, and the large number of experimental studies that have been done with this microorganism.

Fig. 13 presents the results of one measurement channel. It shows the relative shift over time of the resonance frequency and the resistance at resonance with respect to the first measurement (frequency and resistance at the beginning of the experiment).
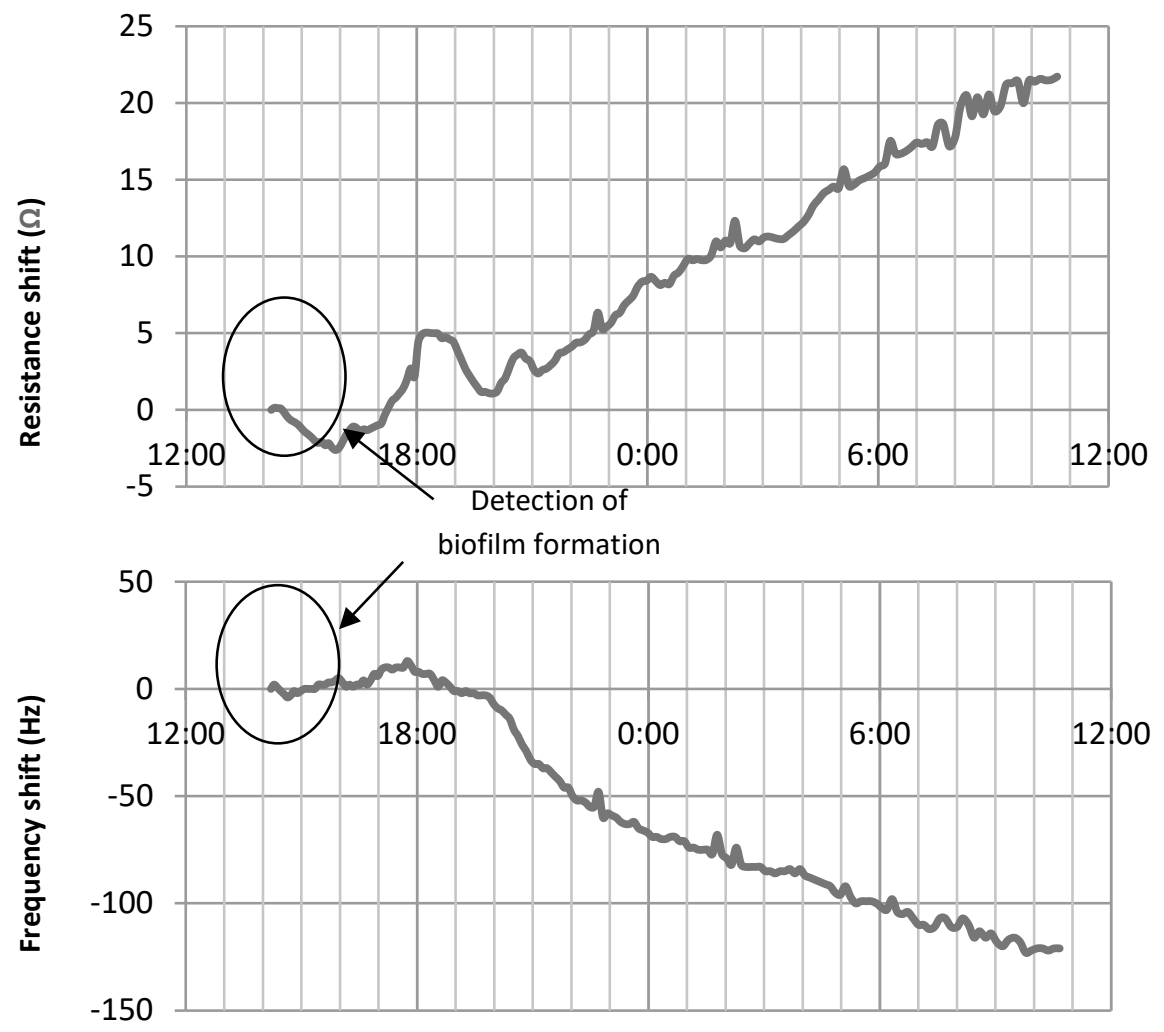

Fig. 13. Resistance at resonance shift (above) and resonance frequency shift (below) of the experiment. 
The obtained results show how the series resonance frequency decreases and the resistance at resonance increases over the time. This means that there are losses in the QCM sensors and they are directly related with the biofilm formation on the crystal surface [11].

\section{Conclusions}

This work describes a new electronic multichannel instrument for continuous monitoring of bacterial cultures in order to know when the cell self-assembly processes produce the biofilm formation and subsequent growth. Also, it is capable to detect the inhibition of these biofilms in the case of the use of biocidal agents such as antibiotics.

Each measurement channel is based on a QCM sensor and the measurement of its electrical impedance to obtain both its series resonance frequency and its resistance at this frequency, which are correlated with the viscoelastic properties of the medium in physical contact with it. This technique allows the bacterial process monitoring without interrupting it unlike other classical techniques where it is necessary to extract a sample of the culture. The use of an auto-balancing impedance bridge, a precise DDS generator of the excitation signal, and a sine wave fitting algorithm to determine the QCM voltage and current, permit a very accurate electrical impedance measurement in an affordable, compact, ease-of-use and portable instrument compared with other alternatives. The commercial Agilent 4294A precision impedance analyser has been used as reference in order to validate the electrical impedance measurements. A $2 \%$ instrument accuracy in the impedance measurement is achieved. Also, the automatic compensation of the QCM static electrical capacitance and the frequency response of the measurement circuit permits to achieve a very accurate determination of resonance parameters in heavily loaded QCM as occurs with biofilms. Results obtained in the measurements of binary mixtures water-glycerol show excellent agreement with the theoretical behaviour demonstrating the effectiveness of this compensation. Experiments with real microorganisms have also demonstrated that the frequency resolution and the impedance accuracy of the instrument permit a qualitative monitoring of the biofilm formation and growth. Although the most relevant information in biofilm processes is obtained with the current instrument, it is expected that it will be able to quantify the thickness of the biofilm. Future works are planned in order to correlate the measurement results of bacteria cultures with the quantity of biofilm adhered to the QCM.

Nevertheless, special attention must be paid to the influence of the temperature in both the bacterial process itself and the signal generation electronic circuits. In order to be able to develop a future commercial prototype, further studies related to the temperature control and its effects in the measurement results in real biofilm samples need to be carried out.

In addition, the scalability and flexibility of the system hardware make it also interesting for new applications requiring on-line electrical impedance analysis or electrical resonance measurement. 


\section{Acknowledgments}

This work was supported by the Ministry of Science, Innovation and Universities of Spain under the project RTI2018-099938-B-I00.

\section{REFERENCES}

[1]. J. Azeredo, N.F. Azevedo, R. Briandet, N. Cerca, T. Coenye, A.R. Costa, M. Desvaux, G. Di Bonaventura, M. Hébraud, Z. Jaglic, M. Kačániová, S. Knøchel, A. Lourenço, F. Mergulhão, R. L. Meyer, G. Nychas, M. Simões, O. Tresse, C. Sternberg, "Critical review on biofilm methods", Critical Reviews in Microbiology, vol. 43, no. 3, pp 313-351, 2017.

[2]. C. Wilson, R. Lukowicz, S. Merchant, H. Valquier-Flynn, J. Caballero, J. Sandoval, M. Okuom, C. Huber, T. Durham Brooks, E. Wilson, B. Clement, C.D. Wentworth, A.E. Holmes, "Quantitative and Qualitative Assessment Methods for Biofilm Growth: A Mini-review", Research \& Reviews: Journal of Engineering and Technology, vol. 6, no. 4, Dec. 2017.

[3]. R.K. Meleppat, C. Shearwood, S.L. Keey, M.V. Matham, "Quantitative optical coherence microscopy for the in situ investigation of the biofilm", J. Biomed. Opt., vol. 21, no. 12, 127002, Dec. 2016.

[4]. C. Larimer, M. Brann, J.D. Suter, R.S. Addleman, "Design of a dynamic biofilm imaging cell for white-light interferometric microscopy", Opt. Eng., vol. 56, no. 11, 111708, Nov. 2017.

[5]. M.U. Farid, J. Guo, A.K. An, "Bacterial inactivation and in situ monitoring of biofilm development on graphene oxide membrane using optical coherence tomography", J. Membr. Sci., vol. 564, pp. 22-34, Oct. 2018.

[6]. N. Zhong, M. Zhao, Y. Li, "U-shaped, double-tapered, fiber-optic sensor for effective biofilm growth monitoring", Biomed. Opt. Express, vol. 7, no. 2, pp. 352-368, Jan. 2016.

[7]. K. Chabowski, A.F. Junka, T. Piasecki, D. Nowak, K. Nitsch, D. Smutnicka, M. Bartoszewicz, M. Moczala, P. Szymczyk, "Impedance Sensors Made in PCB and LTCC Technologies for Monitoring Growth and Degradation of Pseudomonal Biofilm", Metrol. Meas. Syst., vol. 24, no. 2, pp. 369-80, Jun. 2017.

[8]. I. Tubia, J. Paredes, E. Perez-Lorenzo, S. Arana, "Brettanomyces bruxellensis growth detection using interdigitated microelectrode based sensors by means of impedance analysis", Sensors and Actuators, A: Physical, vol. 269, pp. 175-181, Jan. 2018.

[9]. L. Pires, K. Sachsenheimer, T. Kleintschek, A. Waldbaur, T. Schwartz, B.E. Rapp, “Online monitoring of biofilm growth and activity using a combined multi-channel impedimetric and amperometric sensor", Biosensors and Bioelectronics, vol. 47, pp. 157-63, Sept. 2013.

[10].Y. W. Kim, M.P. Mosteller, S. Subramanian, M.T. Meyer, W.E. Bentley, R. Ghodssi, "An optical microfluidic platform for spatiotemporal biofilm treatment monitoring", Journal of Micromechanics and Microengineering, vol. 26, no. 1, 015013, Jan. 2016. 
[11].Y. Zhang, C. Li, Y. Wu, Y. Zhang, Z. Zhou, B. Cao, "A microfluidic gradient mixer-flow chamber as a new tool to study biofilm development under defined solute gradients", Biotechnology and Bioengineering, vol. 116, no. 1, pp. 54-64, Jan. 2019.

[12].T. Piasecki, G. Gula, K. Waszczuk, Z. Drulis-Kawa, T. Gotszalk, “Quartz tuning fork as in situ sensor of bacterial biofilm", Sensors and Actuators B: Chemical, vol. 210, pp. 825-829, Apr. 2015.

[13].G. Gula, K. Waszczuk, T. Olszak, J. Majewska, J. Sarowska, T. Gotszalk, J. Gutowicz, Z.; Drulis-Kawa, “Piezoelectric tuning fork based mass measurement method as a novel tool for determination of antibiotic activity on bacterial biofilm", Sensors and Actuators: B Chemical, vol. 175, pp. 34-9, Dec. 2012.

[14].S. Hohmann, S. Kogel, Y. Brunner, B. Schmieg, "Surface Acoustic Wave (SAW) Resonators for Monitoring Conditioning Film Formation", Sensors, vol. 15, no. 5, pp. 11873-11888, May 2015.

[15].Y. W. Kim; M.T. Meyer, A. Berkovich, S. Subramanian, A.A. Iliadis, W.E. Bentley, R. Ghodssi, "A surface acoustic wave biofilm sensor integrated with a treatment method based on the bioelectric effect", Sensors and Actuators A: Physical, vol. 238, pp. 140-149, Feb. 2016.

[16].S. Krajewski, J. Rheinlaender, P. Ries, D. Canjuga, C. Mack, L. Scheideler, T.E. Schäffer, J. Geis-Gerstorfer, H.P. Wendel, F. Rupp, "Bacterial interactions with proteins and cells relevant to the development of life-threatening endocarditis studied by use of a quartz-crystal microbalance", Anal. Bioanal. Chem., vol. 406, no. 14, pp. 33953406, May 2014.

[17].R. Huang, P. Yi, Y. Tang, "Probing the interactions of organic molecules, nanomaterials, and microbes with solid surfaces using quartz crystal microbalances: methodology, advantages, and limitations", Environmental Science: Processes \& Impacts, vol. 19, no. 6, pp. 793-811, Jun. 2017.

[18].P. Sievers, C. Moss, U. Schroder, D. Johannsmann, "Use of torsional resonators to monitor electroactive biofilms", Biosensors and Bioelectronics, vol. 110, pp. 225-232, Jul. 2018.

[19].D.E. Nivens, J.Q. Chambers, T.R. Anderson, D.C. White, “Long-Term, On-line Monitoring of Microbial Biofilms Using a Quartz Crystal Microbalance", Anal. Chem., vol. 65, no. 1, pp. 65-69, Jan. 1993.

[20].P. Castro, P. Resa, C. Duran, J.R. Maestre, M. Mateo, L. Elvira, “Continuous monitoring of microbial biofilm growth using thickness-shear mode resonators", IOP Conf. Series: Materials Science and Engineering, International Symposium on Ultrasound in the Control of Industrial Processes (UCIP 2012), vol. 42, 012054, 2012.

[21].P. Castro, L. Elvira, J.R. Maestre, F. Montero de Espinosa, "Study of the Relation between the Resonance Behavior of Thickness Shear Mode (TSM) Sensors and the Mechanical Characteristics of Biofilms", Sensors, vol. 17, 1395, 2017.

[22].R.E. Speight, M.A. Cooper, "A Survey of the 2010 Quartz Crystal Microbalance Literature”, J. Mol. Recognit., vol. 25, no. 9, pp. 451-473, Sep. 2012.

[23].S.K. Vashist, P. Vashist, "Recent Advances in Quartz Crystal Microbalance-Based Sensors", Journal of sensors, vol. 2011, Article ID 571405, 13 pages, 2011.

[24].A.L.J. Olsson, M.R. Mitzel, N. Tufenkji, “QCM-D for non-destructive real-time assessment of Pseudomonas aeruginosa biofilm attachment to the substratum during biofilm growth", Colloids and Surfaces B: Biointerfaces, vol. 136, pp. 928-934, Dec. 2015. 
[25].H. Huang, L. Ding, H. Ren, J. Geng, K. Xu, Y. Zhang, "Preconditioning of model biocarriers by soluble pollutants: A QCM-D study", ACS Appl. Mater. Interfaces, vol. 7, no. 13, pp. 7222-7230, Apr. 2015.

[26].A. Arnau (Ed.), Piezoelectric Transducers and Applications, Springer-Verlag, pp 73-74, 2004.

[27].J. Henderson, Electronic Devices. Concepts and Applications, Prentice Hall, NJ, p. 357, 1991.

[28].G. Sauerbrey, "Use of quartz crystal vibrator for weighting thin films on a microbalance", Z Phys., vol. 155, no. 2, pp. 206-222, Apr. 1959.

[29].A.P.M. Glassford, "Response of a Quartz Crystal Microbalance to a liquid deposit", J. Vac. Sci. Technol., vol. 15, no.6, pp. 1836-1843, 1978.

[30].K. K. Kanazawa, J.G Gordon I, "Frequency of a Quartz Microbalance in Contact with Liquid”, Anal Chem., vol. 57, no. 8, pp. 1770-1771, Jul. 1985.

[31].S. Martin, V.E. Granstaff, G.C. Frye, "Characterization of a Quartz Crystal Microbalance with Simultaneous Mass and Liquid Loading", Anal. Chem., vol. 63, no. 20, pp. 2272-2281. Oct. 1991.

[32].M. Schmid, E. Benes, R. Sedlaczek, "A computer-controlled system for the measurement of complete admittance spectra of piezoelectric resonators", Meas. Sci. Technol., vol. 1, no. 9, pp. 970-975, 1990.

[33].J. Schröder, R. Borngräber, F. Eichelbaum, P. Hauptmann, “Advanced interface electronics and methods for QCM", Sensors and Actuators A, vol. 97-98, pp. 543-547, 2002.

[34].R. Schnitzer, C. Reiter, K.C. Harms, E. Benes, M. Gröschl, “A general-purpose online measurement system for resonant BAW sensors", IEEE Sensors Journal, vol. 6, no. 5, pp. 1314-1322, Oct. 2006.

[35].H. Ehahoun, C. Gabrielli, M. Keddam, H. Perrot, P. Rousseau, "Performances and limits of a parallel oscillator for electrochemical quartz crystal microbalances", Anal Chem., vol. 74, no. 5, pp. 1119-1127, 2002.

[36].R. Borngräber, J. Schröder, R. Lucklum, P. Hauptmann, "Is an oscillator-based measurement adequate in a liquid environment?", IEEE Trans. Ultrason. Ferroelect. Freq. Contr., vol. 49, no. 9, pp. 1254-1259, Oct. 2002.

[37].V. Ferrari, D. Marioli, A. Taroni, "Improving the accuracy and operating range of quartz microbalance sensors by purposely designed oscillator circuit", IEEE Trans. Instrum. Meas., vol. 50, no. 5, pp. 1119-1122, Oct. 2001.

[38].A. Arnau, J.V. García, Y. Jiménez, V. Ferrari, M. Ferrari, "Improved Electronic Interfaces for Heavy Loaded at Cut Quartz Crystal Microbalance Sensors", in Proceedings of Frequency Control Symposium Joint with the 21st European Frequency and Time Forum. IEEE International, 2007; pp. 357-362.

[39].M. Ferrari, V. Ferrari, D. Marioli, A. Taroni, M. Suman, E. Dalcanale, "In-liquid sensing of chemical compounds by QCM sensors coupled with high-accuracy ACC oscillator", IEEE Trans. Instrum. Meas., vol. 55, no. 3, pp. 828-834, Jun. 2006.

[40].A. Arnau, "A Review of interface Electronic Systems for AT-cut Quartz Crystal Microbalance Applications in Liquids", Sensors, vol. 8, pp. 370-411, 2008.

[41].K.M. Ibrahim, M.A.H. Abdul-Karim, "A Novel Digital Phase Meter", IEEE Trans. Instrum. Meas., vol. IM-36, pp. 711-716, Sep. 1987.

[42].S. M. Mahmud, "High Precision Phase Measurement Using Adaptive Sampling”, IEEE Trans. Instrum. Meas., vol. 38, no. 5, pp. 954-960, Oct. 1989. 
[43].M. Duric, Z. Durisic, "Combined Fourier and Zero Crossing Technique for Frequency Measurement in Power Networks in the Presence of Harmonics", Renewable Energy \& Power Quality Journal, vol. 1, no. 3, pp. 463-469, Mar. 2005.

[44].R. Micheletti, “Phase Angle Measurement Between Two Sinusoidal Signals”, IEEE Trans. Instrum. Meas., vol. 40, pp. 40-42, Feb. 1991.

[45].M. Sedlacek, "Digital measurement of phase difference - a comparative study of DSP algorithms,", in Proceedings of IMEKO XVII World Congress, June 2003, pp. 639-644.

[46].IEEE Standard for Digitizing Waveform Recorders, IEEE Std. 1057-1994 (R2001), New York, Sep. 2001.

[47].IEEE Standard for Terminology and Test Methods for Analog-to-Digital Converters, IEEE Std. 1241-2000 (R2010), New York, 2010.

[48].T. Radil, P. M. Ramos, A. Cruz-Serra, "Impedance Measurement with Sine-Fitting Algorithms Implemented in a DSP Portable Device”, IEEE Trans. Instrum. Meas., vol. 57, no. 1, pp. 197-204, Jan. 2008.

[49].J.A. Chavez Dominguez, M.J. Garcia Hernandez, O. Millan, I. Tur, A. Turo, M.A. Amer, J. Salazar, “Comparison between Sine Wave Fitting and Zero-Crossing Methods Applied to QCM Impedance Measurements", in Proceedings of the 2017 IEEE International Instrumentation and Measurement Technology Conference (I2MTC), May 2017.

[50].M.A. Amer, J.A. Chavez-Dominguez, M.J. Garcia Hernández, J. Salazar, A. Turo, “Quartz Crystal Microbalance Holder Design for On-Line Sensing in Liquid Applications", International Journal of Electrical, Computer, Energetic, Electronic and Communication Engineering, vol. 10, no. 5, pp. 602-605, 2016. 